\title{
Overview of the CMS detector performance in LHC run II
}

\author{
L. Borrello ${ }^{1, a}$ on behalf of the CMS collaboration \\ ${ }^{1}$ University and INFN Pisa, Italy
}

\begin{abstract}
The CMS experiment at the LHC successfully collected data in 2015 during proton-proton collision at $13 \mathrm{TeV}$ center of mass energy and lead-lead collision at 5.02 $\mathrm{TeV} /$ nucleon. An intense activity has been performed to further improve the detector and the trigger before the LHC restart in 2016. Report on the performance of the trigger, on the commissioning of the main detector components and on the physics objects will be presented based on the latest collected data.
\end{abstract}

\section{Introduction}

The CMS (Compact Muon Solenoid) experiment [1] successfully recorded data during the Large Hadron Collider (LHC) operation in Run 1 which covered 2010-2013 period.

Important physics results have been achieved including the discovery of the Higgs boson announced in July 2012 [2]. Despite the experiment reached this important milestone in the physics program, there are fundamental questions which are not yet answered by the Standard Model. The LHC operation at $13 \mathrm{TeV}$ center of mass energy started in 2015 and opened a new energy domain which should allow to address some of these fundamental questions.

In order to fully exploit the physics potential, major improvements of the CMS experiment has been implemented to cope with the challenging condition of the LHC machine in Run 2. This paper describes the commissioning of the main detector components, the new trigger system and the performance of the physics objects.

\section{Upgrade of the CMS experiment}

During the long shutdown, lasted from March 2013 up to February 2015, the CMS experiment has undergone major changes involving the detector, the data acquisition and the trigger system.

The data acquisition (DAQ) [3] system has been completely redesigned to take the advantage of the latest developments in the computing industry and to cope with the upgraded electronics used for some sub-detectors. In addition, the High Level Trigger (HLT) processing is now file based to allow the DAQ and the HLT systems to be independent. Following the upgrade of the data acquisition system, the online monitoring system used to check the data quality has been completely re-organized.

The Level-1 (L1) trigger system was upgraded to increase the calorimeter tower-level precision reducing the out-of-time pile-up contribution and to combine the candidates from the three muon subdetectors providing a higher efficiency and robustness.

\footnotetext{
a e-mail: laura.borrello@cern.ch
} 
Many activities were done on the detector affecting both the hardware and software. A non exhaustive list include the upgrade of the tracker detector cooling to operate at low temperature, the addition of two new planes of muon chambers to improve the trigger acceptance, the replacement of the readout electronics of the hadron calorimeter and the first upgrade of the trigger system.

Further activities were done during the end of the year technical stop (YETS) mainly to consolidate the cryogenic system of the CMS magnet and to complete the upgrade of the trigger system.

In 2015, the cryogenic system of the CMS solenoid showed some issues and the solenoid could not be operated stably. Hence a detailed plan was performed to refurbish the cryogenic system for stable magnet operation. The cold box of the cryogenic system was cleaned to remove traces of the oil contaminant and the primary oil removal system was fully replaced. After completing the scheduled interventions, the nominal magnetic field of $3.8 \mathrm{~T}$ was reached at the beginning of LHC physics operation and the cryogenic system was stable during 2016.

The CMS L1 trigger architecture upgrade was completed in order to maintain and improve the trigger performance under the extreme LHC operating conditions.

\section{Status of the CMS experiment at the start of 2016}

Before resuming the LHC operation, cosmic data and first collision events were used to perform the commissioning of the trigger system, detectors and to produce the first alignment constants of the Tracker subsystem.

The commissioning period was successful as proved considering the excellent status of the CMS experiment. The detector availability is shown in figure 1 where the fraction of active channel per subsystem in 2016 is compared with the number observed before the technical stop. Status is compa-

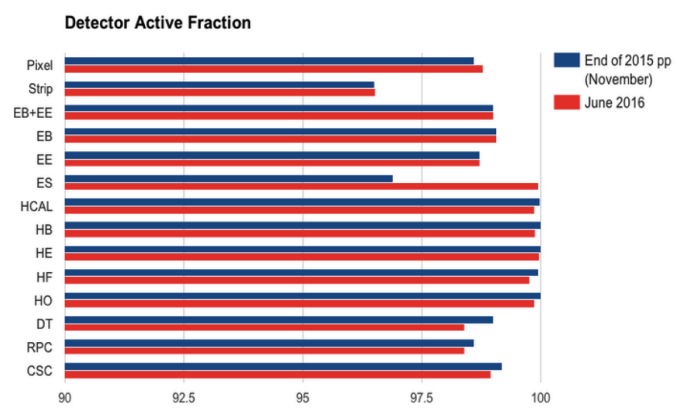

Figure 1: Fraction of active channel operational for each detector

rable for all subsystems while a clear improvement is shown for the preshower detector where $3 \%$ of the channels have been recovered before the intervention on the hardware.

The tracker detector, made of Pixel and Silicon Strip Tracker, is running stably at $-15^{\circ} \mathrm{C}$ since the beginning of Run 2. About 1.5 million cosmic data have been collected to update the alignment constants while first collision data have been used for timing optimization purpose.

The readout system of the electromagnetic calorimeter and the algorithm used for its local reconstruction have been tuned to cope with higher pile-up operation while trigger and readout timing were fully validated. Calibration stream were deployed and first data analysed to update the alignment and intercalibration constants.

The Hadron Calorimeter (HCAL) was improved with new fast readout (10Gb/s links) for barrel and 
endcap system. A new data quality monitoring framework was deployed to improve the detection of data integrity problems. First collision events confirmed the timing synchronization and the good matching between data and trigger primitives.

The muon detectors (DT, CSC, RPC) performed well during first collisions showing for all subsystems good efficiency and a time resolution which is comparable or even better than in 2015 .

The operation of the CMS experiment is summarized in figure 2 where the integrated luminosity

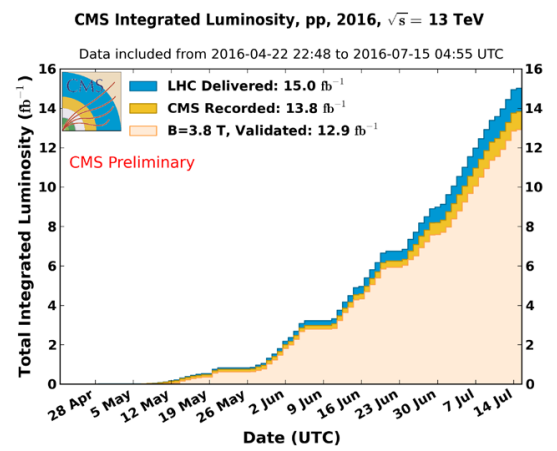

Figure 2: Cumulative curves for the luminosity delivered by LHC (azure), recorded by CMS (orange) and certified as good for physics analysis during stable beams (light orange) for proton-proton collisions at $13 \mathrm{TeV}$ center of mass energy.

delivered by the LHC machine is reported together with the luminosity recorded by the CMS detector and the data validated for physics analysis. The efficiency of data taking and data considered good for physics analysis is higher than $90 \%$ confirming the excellent work done by the CMS collaboration.

\section{Performance of physics object}

The L1 trigger architecture was fully upgraded to maintain and improve the trigger performance in Run 2. First collision data were used to commission the L1 trigger which proved to be effective. Fig-
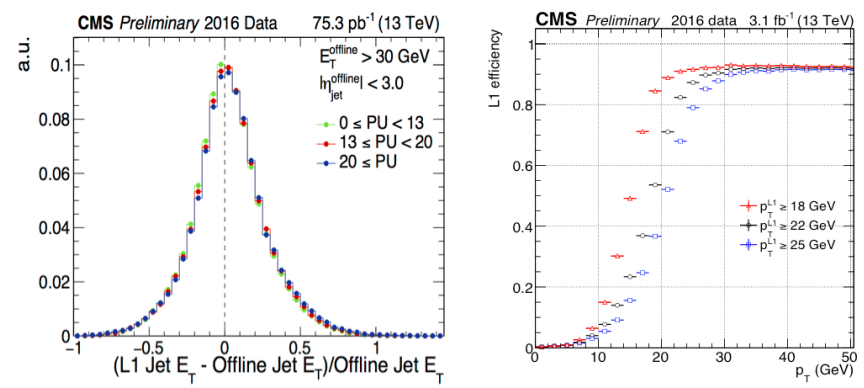

Figure 3: Left: Jet transverse energy resolution for central jets as a function of different pile-up events. Rigth: Trigger efficiency for the single muon path as a function of the offline reconstructed muon transverse momentum

ure 3 (left) shows the difference between Level-1 and offline jet transverse energy for central jets. The 
distribution is split into different pile-up (PU) ranges and the dependency on the number of PU events is negligible. For jets in the forward calorimeter, there is a small dependency on the number of pile up collisions which does not impact the performance significantly.

The L1 muon trigger was redesigned to work with three regional track finders based on the pseudorapidity to exploit the detector redundancy at a lower level. Trigger objects are then merged and cleaned to remove duplicate object by a dedicated global muon trigger. Figure 3 (right) shows the trigger efficiency for the single muon path as a function of the offline reconstructed muon transverse momentum emphasising the turn on part of efficiency. The efficiency is measured with a tag and probe method on a dataset of events recorded using a single muon trigger and the measured value is above $90 \%$.

The electron reconstruction is good as shown in figure 4 where the di-electron invariant mass for

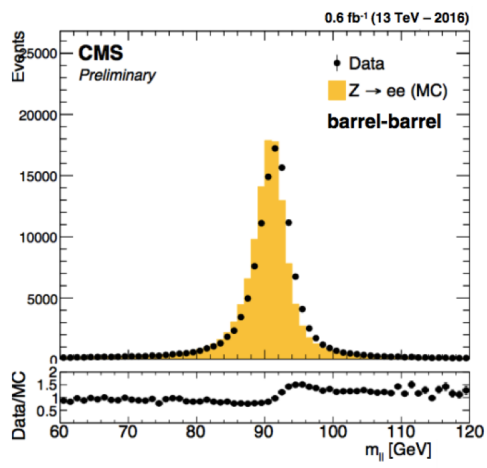

Figure 4: Di-electron invariant mass for electron pairs reconstructed in the barrel region before scale calibration is applied.

electron pairs in the barrel region is reconstructed. An energy shift is observed in data since energy scale and smearing are not applied and the ECAL calibration is not yet updated.

The jet energy response is measured in simulated proton collisions and in data using $\mathrm{Z} / \gamma+$ jet events.
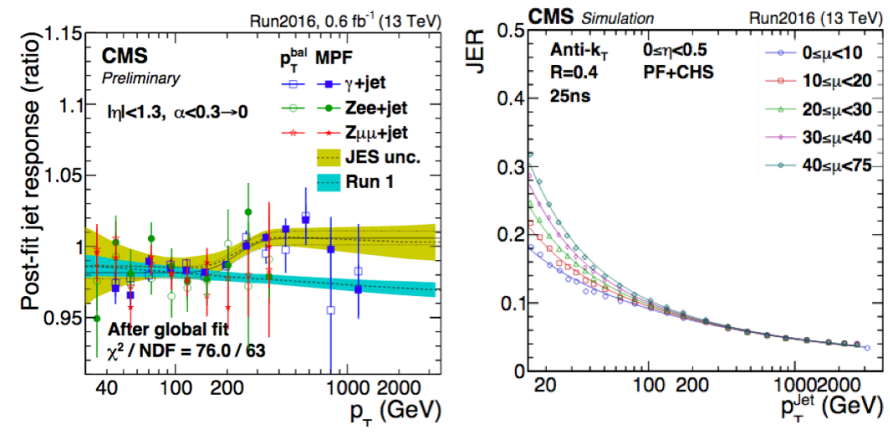

Figure 5: Left: post-fit jet response as a function of the jet transverse momentum. Right: jet energy resolution as a function of the jet transverse momentum for different pile-up events.

The ratio of the data to simulated jet energy response is shown in the left panel of figure 5. The kink observed around $150 \mathrm{GeV}$ is attributed to changes in hadronic calorimeter local reconstruction. 
Performance are similar to Run 1 while the jet energy resolution proved to be robust against pile-up events as shown in the right panel of figure 5.

The performance of the missing transverse energy $\left(\mathrm{E}_{T}^{\text {miss }}\right)$ were studied in events with an identified
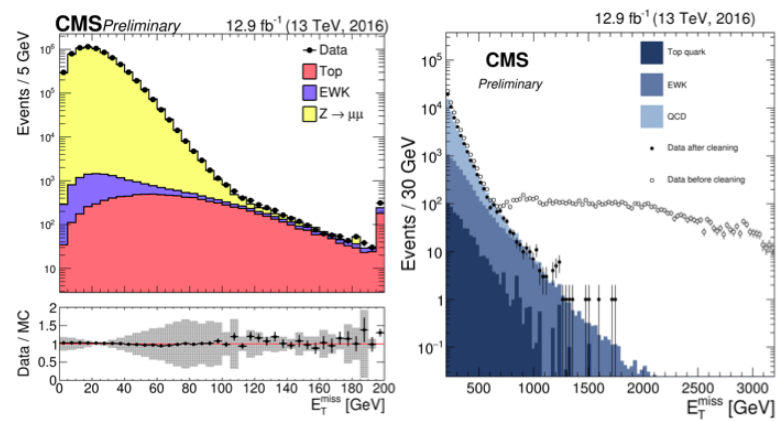

Figure 6: Left: Distributions of $\mathrm{E}_{T}^{\text {miss }}$ in $Z \rightarrow \mu^{+} \mu^{-}$events. The points in the lower panel show the data/MC ratio, including the statistical uncertainties. The last bin contains overflow content. Right: The $\mathrm{E}_{T}^{\text {miss }}$ distributions for events passing the dijet selection after applying the cleaning algorithms.

$\mathrm{Z}$ boson or an isolated photon. The distribution of $\mathrm{E}_{T}^{\text {miss }}$ for events passing a $Z \rightarrow \mu \mu$ selection is shown in figure 6 where is evident the good agreement between data and simulation. The right plot in figure 6 shows the good result of the cleaning procedure applied to remove the long MET tail which is produced by different phenomena in the calorimeters.

Also the high-level object identification algorithms have been reoptimized for Run 2 . The hadronic $\tau$ decays is well identified as shown in the left panel of figure 7. The improved MVA-based identifica-
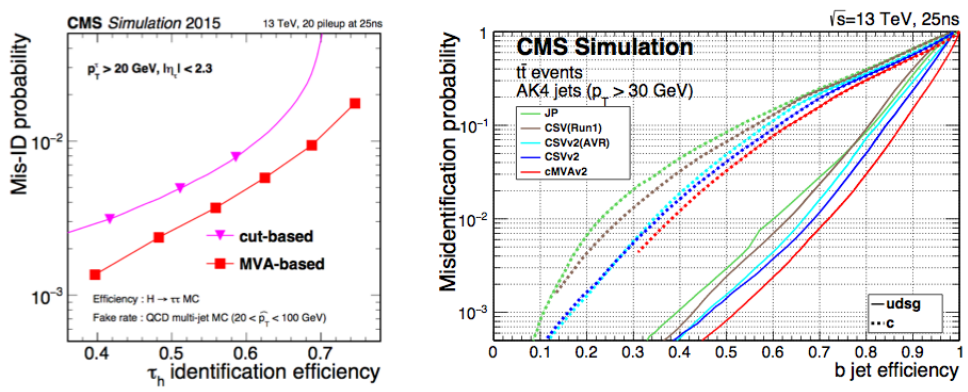

Figure 7: Left: misidentification probability as a function of the hadronic $\tau$ identification efficiency in multijet events (for the background) and Higgs decays to two $\tau$ leptons (for the signal) at $13 \mathrm{TeV}$. Rigth: Misidentification probability for $\mathrm{c}$ and $\mathrm{d}, \mathrm{u}, \mathrm{s}, \mathrm{g}$ jets as a function of the $\mathrm{b}$ jet identification efficiency in $t \bar{t}$ events at $13 \mathrm{TeV}$.

tion efficiency is performing significantly better than the cut-based strategy, with a $20 \%$ difference in relative efficiency.

Right panel of figure 7 shows the performance of different algorithms compared with the Combined Secondary Vertex (CSV) used in Run 1. The comparison highligths the better performance of the algorithms which have been modified for Run 2 operation. As an example, the CSVv2 algorithm, 
which combines the secondary vertex and track-based lifetime information with a neural network instead of a likelihood ratio, provides an identification probability which is about $10 \%$ higher than the value obtained with the previous algorithm.

\section{Conclusions}

We discussed the activity done in the CMS experiment in preparation of LHC Run 2 operation and presented the performance of the detector and the physics object at the start of 2016.

The CMS detector is running stably with excellent performance and the collaboration is ready to exploit the full physics potential collecting and analyzing the large datasets provided by the LHC.

\section{References}

[1] CMS Collaboration, JINST 33 S08004 (2008).

[2] S. Chatrchyan et al. (CMS collaboration), Phys.Lett. B716, 30-61 (2012)

[3] T. Bawej et al., IEEE Transactions on Nuclear Science, VOL. 62, NO. 3, 1099, (2015) 